\title{
Shot noise in an anyonic Mach-Zehnder interferometer
}

\author{
D. E. Feldman, ${ }^{1}$ Yuval Gefen, ${ }^{2}$ Alexei Kitaev, ${ }^{3,4}$ K. T. Law, ${ }^{1}$ and Ady Stern ${ }^{2}$ \\ ${ }^{1}$ Department of Physics, Brown University, Providence, Rhode Island 02912, USA \\ ${ }^{2}$ Condensed Matter Physics Department, Weizmann Institute of Science, Rehovot 76100, Israel \\ ${ }^{3}$ California Institute of Technology, Pasadena, California 91125, USA \\ ${ }^{4}$ Microsoft Project Q, University of California, Santa Barbara, California 93106, USA
}

(Received 6 July 2007; published 21 August 2007)

\begin{abstract}
We show how shot noise in an electronic Mach-Zehnder interferometer in the fractional quantum Hall regime probes the charge and statistics of quantum Hall quasiparticles. The dependence of the noise on the magnetic flux through the interferometer allows for a simple way to distinguish Abelian from non-Abelian quasiparticle statistics. In the Abelian case, the Fano factor (in units of the electron charge) is always lower than unity. In the non-Abelian case, the maximal Fano factor as a function of the magnetic flux exceeds 1.
\end{abstract}

DOI: 10.1103/PhysRevB.76.085333

\section{INTRODUCTION}

Among the most fascinating features of the quantum Hall effect (QHE) are the fractional charge and statistics of elementary excitations. The charge of a quasiparticle is a simple fraction of an electron charge. In most QHE states, the quasiparticles are Abelian anyons, i.e., they accumulate nontrivial phases when encircling each other. For some filling factors, even more interesting non-Abelian statistics was predicted. ${ }^{1,2}$ When a single non-Abelian anyon makes a full circle around another (identical) one, this not only modifies the phase of the wave function, but can also result in a change of the quantum state of the quasiparticle system. Such a property makes non-Abelian anyons promising for fault-tolerant quantum computation. ${ }^{3,4}$

Detecting fractional charge and statistics proved to be a difficult task. Fractional charges were eventually observed in shot noise experiments at Weizmann Institute and Saclay. ${ }^{5}$ No nonambiguous observation of the statistics of identical fractional quasiparticles has been reported so far in spite of several theoretical proposals for detecting Abelian ${ }^{6-10}$ and non-Abelian ${ }^{10-16}$ anyons, and important efforts in this direction. ${ }^{17,18}$ The setup, ${ }^{5}$ which was successful for measuring fractional charges via shot noise, cannot be applied to the problem of fractional statistics since no quasiparticle braiding occurs in such a device.

Recently a different device, an electronic Mach-Zehnder interferometer, has been designed and fabricated at Weizmann Institute ${ }^{19}$ (Fig. 1). Quasiparticle braiding is possible in that configuration. As we show below, shot noise in the Mach-Zehnder interferometer contains information about both charge and statistics of the elementary excitations. In particular, it allows for a simple and physically transparent way to distinguish Abelian from non-Abelian quasiparticles.

Anyon statistics is defined in terms of quasiparticle braiding. Hence, interference experiments may be a useful tool for experimentally observing it. It has been proposed to employ electronic Fabry-Pérot and Mach-Zehnder interferometers for probing Abelian ${ }^{6,9}$ and non-Abelian ${ }^{11-14,16}$ statistics. The Fabry-Pérot approach is simple and transparent: it is based on quasiparticles moving along the edge, encircling quasiparticles localized in the bulk. This approach
PACS number(s): 73.43.Jn, 05.40.Ca, 73.43.Cd, 73.43.Fj

is, however, sensitive to fluctuations of the number of trapped quasiparticles $8,13,20$ which can tunnel to and from localized states inside the interferometer. In the absence of these fluctuations, the interference pattern that results from the lowest-order backscattering amplitude shows clear and unambiguous signatures of Abelian and nonAbelian statistics. When such fluctuations are present and are slow compared to the scale of the particle time of flight through the interferometer, lowest-order interference is wiped out. The remaining signal, originating from multiple reflections, shows less striking signatures in the visibility of the interference. An additional signature is seen, in this case, in current noise. ${ }^{10}$ The Mach-Zehnder interferometer is not sensitive to these slow fluctuations, ${ }^{9,16}$ and shows signatures of Abelian and non-Abelian statistics in the visibility of the interference and in the $I-V$ characteristics. Fast fluctuations suppress the interference in the two geometries. In both cases, the experimental outcome depends on unknown nonuniversal microscopic parameters. Obviously, it would be desirable to find a simpler procedure based on a single measurement of a universal quantity. We show below that this can be accomplished in a shot noise experiment with an anyonic Mach-Zehnder interferometer. The information about statistics is contained in a single universal quantity: the maximal Fano factor at low temperatures.

Nonequilibrium noise is an important experimental tool in mesoscopic physics. We define it as the Fourier transform of the current-current correlation function, ${ }^{21}$

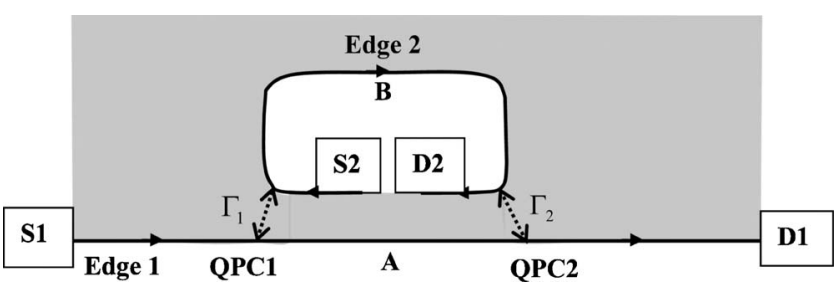

FIG. 1. Schematic picture of the anyonic Mach-Zehnder interferometer. Arrows indicate the edge mode propagation directions from source S1 to drain D1 and from source S2 to drain D2. 


$$
S(\omega)=\frac{1}{2} \int_{-\infty}^{\infty}\langle\hat{I}(0) \hat{I}(t)+\hat{I}(t) \hat{I}(0)\rangle \exp (i \omega t) d t
$$

It is often convenient to express the noise in terms of an effective charge $e^{*}(\omega)$, according to $S=e^{*}\langle\hat{I}\rangle$. The dimensionless ratio $e^{*}(\omega) / e$ is known as the Fano factor and depends on the temperature, voltage, frequency, disorder, and interaction strength. In some situations, $e^{*}$ is universal and equals the carrier charge. This property was used for probing fractional charges in Ref. 5. Shot noise in the Mach-Zehnder interferometer in the integer QHE has recently attracted much attention. ${ }^{22}$ Below we show that the physics of noise is even richer in the fractional QHE regime. We find that the Fano factor in the Mach-Zehnder interferometer depends on frequency. At high frequencies, the zero-temperature Fano factor is equal to the dimensionless quasiparticle charge. At low frequency, the Fano factor depends on the magnetic flux. The maximum value of the zero-temperature Fano factor is always below 1 in Abelian systems and can exceed 1 for non-Abelian anyons.

Figure 1 shows the structure of the interferometer. ${ }^{19}$ In the absence of interedge tunneling, charge propagates along two chiral edges (S1 to D1 and S2 to D2); the source S1 is kept at a finite voltage $V$ with respect to S2. Quasiparticles can tunnel between the edges at two point contacts QPC1 and QPC2. The current that flows between the edges (i.e., from S1 to D2) depends on $V$, the magnetic flux $\Phi$ through the loop A-QPC2-B-QPC1-A, and the tunneling amplitudes, $\Gamma_{1}$ and $\Gamma_{2}$, at the two quantum tunneling contacts. We assume that the tunneling amplitudes are small. Hence, the mean time between two consecutive quasiparticle tunneling events is much longer than the duration of such an event. We also assume that the leads fully absorb edge excitations. For a system of bosons or fermions, such an assumption implies that tunneling events are independent. In contrast, we will see that the probability of an anyon to tunnel is affected by the history of previous tunneling events.

Below we calculate the noise for the tunneling current between the edges. We will distinguish the high- and lowfrequency regimes. In the low-frequency regime, the frequency $\omega$ is less than $1 / \tau_{T}=I / q$, where $\tau_{T}$ is the typical time interval between consecutive tunneling events, $I$ the average current, and $q$ the quasiparticle charge. In the high-frequency regime, $1 / \tau_{J}>\omega>1 / \tau_{T}$, where $\tau_{J}$ is the time of one tunneling event. The time $\tau_{J} \sim \tau_{\text {travel }}+\tau_{\text {uncertainty }}$ can be estimated as the sum of two contributions. The quasiparticle travel time between the point contacts, $\tau_{\text {travel }}$, is of the order of $\sim L / v$, where $L$ is the interferometer size and $v$ the quasiparticle's velocity along the edges. The time $\tau_{\text {uncertainty }}$ $\sim \hbar / \max \left(q V, k_{B} T\right)$, where $V$ is the voltage bias and $T$ the temperature, comes from the uncertainty of the energy of tunneling quasiparticles.

Most of this paper focuses on the low-frequency noise at zero temperature and on the finite-temperature noise at arbitrary frequency. The zero-temperature high-frequency limit is much simpler. At high frequencies, the contribution to Eq. (1) from large times $t \gg 1 / \omega$ is suppressed by the oscillating exponential factor. The contribution from short times $t \sim \tau_{J}$ can be found by substituting $\omega=0$. One gets $S$ $=\left\langle\hat{I}(0) \int_{-\tau_{J}}^{\tau_{J}} d t \hat{I}(t)\right\rangle=e^{*} I$, where the Fano factor $e^{*}=\int_{-\tau_{J}}^{\tau_{J}} I(t) d t$ equals the charge transmitted during one tunneling event. Thus, in the high-frequency limit, $e^{*}$ is the quasiparticle charge $q$. This result is similar to the low-frequency behavior of the noise in the geometry with one tunneling contact, ${ }^{23}$ and holds for any quasiparticle statistics. In contrast, the lowfrequency noise in the Mach-Zehnder interferometer shows a unique behavior which will allow detecting fractional statistics.

This paper has the following structure. Section II contains the main results of the paper. It focuses on the low-frequency noise at $T=0$ in two types of QHE states: Laughlin states with filling factors $\nu=1 / m$ ( $m$ odd) and the Pfaffian state ${ }^{1,2}$ at filling factor $\nu=5 / 2$. The technical approach of Sec. II is rather simple, and its results can be understood from a simple qualitative picture. The rest of the paper focuses on the finite-temperature noise for arbitrary fractional statistics and is more technical. The method is based on the algebraic theory of anyons (Ref. 4). Section III contains the calculation of the tunneling probabilities. In Sec. IV, we find the tunneling current. In Sec. V, we calculate the zero-frequency noise at arbitrary temperatures. Sec. VI discusses the highfrequency noise with the emphasis on the limit of low voltages and finite temperatures, i.e., the linear response regime. In that regime, the noise and conductance are related by the Nyquist formula. We find that, in contrast to the shot noise, the thermal Nyquist noise is independent of the magnetic flux through the interferometer for any fractional statistics with nondegenerate braiding.

\section{SHOT NOISE AT ZERO TEMPERATURE}

In this section, we consider the Laughlin and Pfaffian states. We first briefly review the anyon statistics in these two states and then calculate the noise.

\section{A. Fractional statistics in Laughlin and Pfaffian states}

The quasihole charge in a Laughlin state with the filling factor $\nu$ is $q=\nu e$. When one quasihole encircles $n$ others counterclockwise, the wave function accumulates the statistical phase

$$
\theta_{s}=2 \pi \nu n
$$

Since the phase $\theta_{s}$ is defined $\bmod 2 \pi$, there are $r$ $=0, \ldots, 1 / \nu-1$ "equivalence classes" which correspond to $1 / \nu$ physically distinct statistical phases $\theta_{s}$. A configuration of $n$ quasiholes belongs to the $r$ th class, $r=n \bmod 1 / \nu$.

In the $\nu=5 / 2$ Pfaffian liquid, quasiholes carry charge $e / 4$. Due to the non-Abelian statistics, the quantum state of a system of several charge-e/4 quasiholes is not uniquely determined by their coordinates. One needs to specify additional quantum numbers, most importantly, the topological charge (for a review, see Ref. 4). In the $\nu=5 / 2$ state, the latter takes on one of three values ${ }^{14}$ : 1 ("vacuum"), $\epsilon$ ("fermion"), and $\sigma$ ("vortex"). In a system with an odd number of quasiholes $n$, the topological charge is always $\sigma$. At even $n$, two topological charges, 1 and $\epsilon$, are possible. When two sets 
of quasiholes merge, the topological charge of the composite system is given by the fusion rules: $\epsilon \times \epsilon=1, \epsilon \times \sigma=\sigma, \sigma$ $\times \sigma=1+\epsilon$. In a process in which a quasihole encircles a set of $n$ quasiholes whose topological charge is $\alpha$, the wave function picks up a statistical phase which depends on $n, \alpha$, and the topological charge $\beta$ of the whole system consisting of $(n+1)$ quasiholes. We denote this statistical phase by $\phi_{a b}$, where $a=(n, \alpha)$ and $b=(n+1, \beta)$ label superselection sectors. ${ }^{4}$ It equals

$$
\phi_{a b}=n \pi / 4+\phi_{\alpha \beta}^{\prime},
$$

where the non-Abelian part $\phi_{\alpha \beta}^{\prime}$ satisfies the following rules: $\phi_{1 \sigma}^{\prime}=0, \phi_{\epsilon \sigma}^{\prime}=\pi, \phi_{\sigma 1}^{\prime}=-\pi / 4$, and $\phi_{\sigma \epsilon}^{\prime}=3 \pi / 4$. As we will see, this information determines the quasihole tunneling rates. The statistical phase factor $\exp \left(i \phi_{a b}\right)$ [Eq. (3)], is unchanged if the state $a=(n, \alpha)$ is fused with an electron whose superselection sector is $(4, \epsilon)$. Thus, the superselection sectors form six equivalence classes, which are characterized by $(n \bmod 4, x)$, where $x=\sigma$ for odd $n$ and either 1 or $\epsilon$ for even $n$.

Due to the energy gap for bulk excitations, the low-energy physics of the Mach-Zehnder interferometer is determined by the edges. Hence, the Hamiltonian is

$$
\hat{H}=\hat{H}_{\text {edge }}+\left[\left(\Gamma_{1} \hat{X}_{1}+\Gamma_{2} \hat{X}_{2}\right)+\text { H.c. }\right],
$$

where $\hat{H}_{\text {edge }}$ is the Hamiltonian of the two edges and the operators $\hat{X}_{1}, \hat{X}_{2}$ correspond to the quasihole transfer from outer edge 1 to inner edge 2 at QPC1 and QPC2, respectively. Strictly speaking, the Hamiltonian must include operators which describe tunneling of the objects with all possible electric and topological charges. We take into account only the quasihole tunneling operators since they are most relevant. ${ }^{24}$ In the limit of small tunneling amplitudes $\Gamma_{1}$ and $\Gamma_{2}$, the probability of a tunneling event from edge 1 to edge 2 includes four contributions: two proportional to $\left|\Gamma_{1,2}^{2}\right|$ (independent of the flux $\Phi$ through the interferometer) and two $\propto \Gamma_{1} \Gamma_{2}^{*}$ and $\propto \Gamma_{1}^{*} \Gamma_{2}$ (flux dependent). The last two contributions describe the interference of quasihole trajectories S1QPC1-B-D2 and S1-A-QPC2-D2, and include the AharonovBohm phase $2 \pi q \Phi /\left[e \Phi_{0}\right]$. Here, $q$ is the quasiparticle charge and $\Phi_{0}=e h / c$ the flux quantum.

Furthermore and central to our discussion is the dependence of these interference terms ${ }^{9,16}$ on the topological charge of edge 2. In the case of the Laughlin states, the tunneling probability depends on the statistical phase Eq. (2), where $n q$ is the total charge transferred between the edges (negative $n$ corresponds to tunneling events from edge 2 to edge 1). In the Pfaffian state, these terms contain the phase (3), where $n$ has the same meaning as in the Abelian case, and $\alpha$ and $\beta$ are the initial and final topological charges of edge 2 . In both cases, a given tunneling event modifies the tunneling rate of the subsequent event. Quasiparticle tunneling between the edges can be viewed as a Markov process. ${ }^{9}, 10,16$

\section{B. Abelian Laughlin case: $\boldsymbol{\nu}=\mathbf{1} / \mathrm{m}$}

The tunneling rates were computed in Ref. 9 (see also Sec. III). Transitions are possible for the states connected by a)

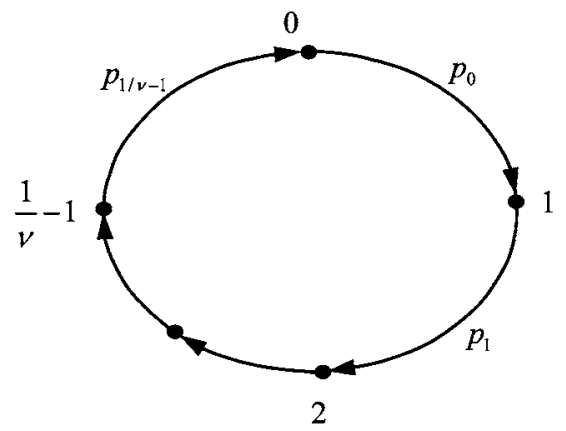

b)

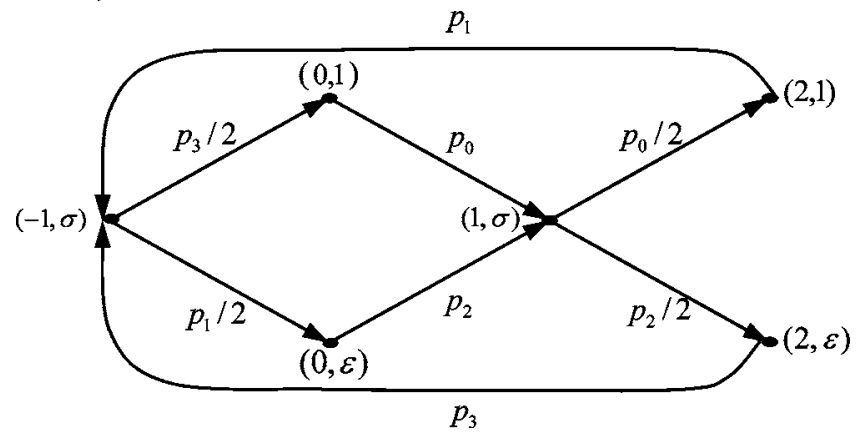

FIG. 2. (a) States and transition probabilities in a Laughlin liquid with the filling factor $\nu$. (b) In the $\nu=5 / 2$ QHE liquid, each state of the interferometer is labeled by the topological charge of edge 2 and the number of the previous tunneling events mod 4 .

arrows in Fig. 2(a). At zero temperature, quasiholes tunnel only from the edge with higher chemical potential (edge 1) to the edge with the lower potential, i.e., only in the direction of the arrows. The precise expressions for the transition rates depend on the edge state model. ${ }^{9}$ We are concerned only with manifestations of fractional statistics and discuss below only those properties of the transition rates which depend on statistics. The transition rate depends ${ }^{9}$ on the number of the previous tunneling events $\bmod m$. Thus, at zero temperature and low voltage, there are $m$ different tunneling rates ${ }^{9}$

$$
\begin{aligned}
p_{k}= & r_{0}(V)\left\{\left|\Gamma_{1}\right|^{2}+\left|\Gamma_{2}\right|^{2}+\left[u(V) \Gamma_{1}^{*} \Gamma_{2} \exp \left(2 \pi i \nu\left[\Phi / \Phi_{0}+k\right]\right)\right.\right. \\
& + \text { c.c. }]\},
\end{aligned}
$$

where $k=0, \ldots, m-1$, and the coefficients $r_{0}$ and $u$ are expressed via the matrix elements of the operators $\hat{X}_{1,2}$. The above perturbative expressions for the rates are valid ${ }^{9,16}$ for such small $\Gamma_{1,2}$ that $p_{k} \ll q V / h$ for all $k$. The coefficient $u$ depends on the voltage and the distance between the tunneling contacts. For $q V L / h v \ll 1$, it follows that $u \rightarrow 1$ (here, $L$ is the interferometer size and $v$ is the edge velocity). If also $\left|\Gamma_{1}\right| \approx\left|\Gamma_{2}\right|$, it is possible to tune the magnetic flux in such a way that one of the rates becomes much smaller than all others.

Let us now calculate the Fano factor. The average square of the time between two subsequent tunneling events is $\overline{t_{k}^{2}}$ $=2 / p_{k}^{2}$. The noise (1) equals $S=\overline{\delta Q^{2}(t)} / t$, where $\delta Q(t)$ is the fluctuation of the charge $Q(t)$ transmitted during the time $t \gg 1 / p_{k}$. The Fano factor reads as 


$$
e^{*}=S / I=\overline{\delta Q^{2}(t)} / \overline{Q(t)} .
$$

The charge transmitted during $N / \nu \gg 1$ tunneling events is $N e$. The average time $t_{N}$ needed for $N / \nu$ tunneling events is $\bar{t}_{N}=N \sum_{k=0}^{(1 / \nu)-1} \frac{1}{p_{k}}$. The time $t_{N}=\bar{t}_{N}+\delta t$ fluctuates. One can estimate $\overline{Q\left(\bar{t}_{N}\right)}$ as $N e$, with the accuracy of $O\left(\delta t / t_{N}\right)$. Such accuracy is insufficient for the calculation of $\overline{\delta Q^{2}\left(\bar{t}_{N}\right)}$. For the sake of evaluating the second moment of the current, one can either calculate the fluctuations of $Q$ during $\bar{t}_{N}$ or assume a uniform current with fluctuations of the time interval $\delta t$. We, thus, need to find the fluctuation $\overline{\delta t^{2}}$. From the expression for $\overline{t_{k}^{2}}$, we find $\overline{\delta t^{2}}=N \Sigma_{k} \frac{1}{p_{k}^{2}}$. Now we can estimate $Q(\bar{t}) \approx N e$ $-\delta t I=$ const $-\delta t N e / \bar{t}_{N}$. Hence, $\overline{\delta Q^{2}}=I^{2} \overline{\delta t^{2}}$. Finally,

$$
e^{*}=e \frac{\sum_{k=0}^{(1 / \nu)-1}\left(1 / p_{k}^{2}\right)}{\left[\sum_{k=0}^{k=(1 / \nu)-1}\left(1 / p_{k}\right)\right]^{2}} .
$$

Since the rates $p_{k}$ depend on the magnetic flux $\Phi$, the lowfrequency Fano factor depends on flux as well, in contrast to the one-point-contact geometry. ${ }^{23}$ From the inequality of arithmetic and quadratic means, we find that $e^{*} \geqslant \nu e$. The maximum value of $e^{*}$ is the electron charge $e$, and it is obtained when one of the transition rates is much smaller than all others (see discussion below).

\section{Shot noise in non-Abelian Pfaffian state}

In the Pfaffian state, there are eight transition rates ${ }^{16}$ [see Fig. 2(b); a general approach to the calculation of transition rates is discussed in Sec. III]. At zero temperature and low voltage, four of them have the form ${ }^{16}$

$$
p_{k}=r_{0}\left\{\left|\Gamma_{1}\right|^{2}+\left|\Gamma_{2}\right|^{2}+\left[u \Gamma_{1}^{*} \Gamma_{2} \exp \left(\pi i\left[\Phi / \Phi_{0}+k\right] / 2\right)+\text { c.c. }\right]\right\},
$$

where $k=0,1,2$, and 3 . The four remaining rates are given by $p_{k} / 2$ [Fig. 2(b)] As in the Laughlin state, if $\left|\Gamma_{1}\right| \approx\left|\Gamma_{2}\right|$ and $u \approx 1$, it is possible to tune the magnetic flux in such a way that one of the rates becomes much smaller than all others. The condition $u \approx 1$ corresponds to close point contacts, i.e., the distances between the point contacts along both edges must be shorter than all other scales: the edge lengths; the scale $h v / q V$, controlled by the voltage bias; and $h v / k_{B} T$, where $T$ is the temperature.

Using the same approach as in the Abelian case, one can rewrite Eq. (6) as

$$
e^{*}=e \overline{\delta t^{2}} /(\bar{t})^{2},
$$

where $\bar{t}$ is the average time needed for four consecutive tunneling events, and $\delta t$ is the fluctuation of that time. Since one electron charge is transferred between the edges during four tunneling events, the current reads as

$$
I=e / \bar{t}
$$

A straightforward calculation yields

$$
\bar{t}=2\left[\sum_{k=0}^{3} \frac{p_{k}}{p_{k+1}}+4\right] /\left[\sum_{k=0}^{3} p_{k}\right]
$$

and

$$
\overline{\delta t^{2}}=\left[\frac{4}{\sigma_{k=0}^{3}} \frac{p_{k}+p_{k+1}}{p_{k+1}^{2}}-\frac{4}{\sigma^{2}}\left(\sum_{k=0}^{3} \frac{p_{k}}{p_{k+1}}\right)^{2}\right],
$$

where $\sigma=\sum p_{k}$ and the convention $p_{3+1}=p_{0}$ is used. The calculation is based on Fig. 2(b). In the space of states, there are four possible trajectories which begin and end in point $(-1, \sigma) . \bar{t}$ and $\overline{t^{2}}$ are combinations of the contributions from the four trajectories weighted according to their probabilities. For example, the probability of the trajectory $(-1, \sigma)$ $\rightarrow(0,1) \rightarrow(1, \sigma) \rightarrow(2,1) \rightarrow(-1, \sigma) \quad$ is $\quad\left[p_{3} /\left(p_{1}+p_{3}\right)\right]$ $\times\left[p_{0} /\left(p_{0}+p_{2}\right)\right]$. In order to make Eqs. (11) and (12) more compact and symmetric, we used the fact that $p_{0}+p_{2}=p_{1}$ $+p_{3}$, which is evident from Eq. (8).

Equations (9)-(12) have an interesting feature: in contrast to the Abelian case [Eq. (7)], the Fano factor can exceed one electron charge. A convenient reference point corresponds to such magnetic flux $\Phi$ where the current (10) is minimal. At $u \approx 1$ and $\Gamma_{1} \approx \Gamma_{2}$, the currents (10) and (11) are minimal for $\Phi=(4 n-k+2) \Phi_{0}$, in which case the tunneling rate $p_{k}[\mathrm{Eq}$. (8)] is close to zero. Assuming $k=0$, one gets $p_{0} \ll p_{1}, p_{2}, p_{3}$. Then Eqs. (8)-(12) imply that $e^{*}=3 e>e$. The maximal Fano factor is achieved at a different value of the magnetic flux. It can be found numerically from Eqs. (9), (11), and (12). In the limit $u \approx 1$ and $\Gamma_{1} \approx \Gamma_{2}$, the maximal effective charge equals $e_{\max }^{*} \approx 3.2 e$. In contrast, the maximal value of the Fano factor of the Laughlin state [Eq. (7)] is $e^{*}=e$.

The difference between the two cases provides a way to distinguish between Abelian and non-Abelian statistics from shot noise. In fact, this difference may be understood from Fig. 2 without detailed calculations. Both in the Abelian and non-Abelian cases, the minimal current corresponds to $p_{0}$ $\approx 0$. Transport in a Laughlin state can be described in the following way: typically, the system stays in state 0 [Fig. $2(\mathrm{a})]$ for a long time, after which it tunnels to state 1 . From state 1, it quickly goes through the series of states $2,3, \ldots, m-1$ and back to 0 . Then the system stays in state 0 again for a long time. Thus, charge is typically transmitted in "bursts" of $m$ quasiparticles, carrying altogether one electron charge. This gives $e^{*}=e$. In the non-Abelian case [Fig. 2(b)] the transport also occurs rapidly between prolonged stays in state $(0,1)$. However, the average charge transmitted between two such stays exceeds an electron charge due to the existence of a "bypass road" $(-1, \sigma) \rightarrow(0, \epsilon) \rightarrow(1, \sigma) \rightarrow(2, \epsilon)$ $\rightarrow(-1, \sigma)$.

\section{TRANSITION RATES}

In this section, we calculate transition rates for arbitrary fractional statistics at arbitrary temperature $T$ and voltage $V$. We assume that the topological charge of elementary excitations has a finite number of possible values. There is an infinite number of possible electric charges. We will denote the minimal charge of an anyon as $q>0$. The quantum di- 
mensions $d_{a}$, fusion multiplicities $N_{a x}^{b}$, and statistical phases $\phi_{a b}$ [Eq. (13)] depend only on the topological charges. All these conditions are satisfied for the Laughlin and Pfaffian statistics for an appropriate definition of the topological charge: Each vertex of the graphs in Fig. 2 should be viewed as a class of states with the same topological charge, and different topological charges should be ascribed to different vertices.

We will work in the limit of small $\Gamma_{1}$ and $\Gamma_{2}$ [Eq. (4)], so that the transition rates can be found from the lowest, second, order in $\Gamma$ 's. Physically, this means that at every moment of time there is no more than one quasiparticle between the point contacts. Nevertheless, the transition rates depend on all previous tunneling events through the total topological charge of the edges.

Consider a tunneling event in which a quasiparticle with electric charge $q$ and topological charge $x$ is transferred from the outer edge 1 to the inner edge 2 (Fig. 1). After such an event, the topological charge of edge 2 changes. We will denote the initial topological charge of edge 2 as $a$ and the final topological charge as $b$. Possible values of $b$ are determined by the fusion rules for charges $x$ and $a$. Since the total topological charge of the interferometer must remain at the vacuum value 1 , the topological charge of edge 2 is always opposite to the topological charge of edge 1. In what follows, we will use the notation $\bar{a}$ for the topological charge opposite to $a$.

The tunneling operators $\hat{X}_{1}$ and $\hat{X}_{2}$ [Eq. (4)] depend on the gauge. The choice of the vector potential $\vec{A}$ is restricted by the condition that its circulation over the loop A-QPC2-BQPC1-A (Fig. 1) equals the magnetic flux $\Phi$ through the interferometer. At the most convenient choice of the gauge, the vector potential is nonzero only inside the point contact QPC2. In this case, the edge Hamiltonian and the tunneling operator $\hat{X}_{1}$ are independent of the magnetic flux, and the operator $\hat{X}_{2}$ contains the factor $\exp \left(i \phi_{\mathrm{AB}}\right)$, where the Aharonov-Bohm (AB) phase $\phi_{\mathrm{AB}}=2 \pi i q \Phi / e \Phi_{0}$ and $\Phi_{0}$ $=h c / e$ is the flux quantum.

In addition to the Aharonov-Bohm phase, a particle accumulates a statistical phase $\phi_{a b}$ when it goes along the loop A-QPC2-B-QPC1-A. Hence, $\hat{X}_{2}$ contains an additional factor $\exp \left(i \phi_{a b}\right)$. For the calculation of the statistical phase, we consider a process in which a particle with topological charge $x$ encircles the hole in the interferometer (Fig. 1) which contains topological charge $a$. The result of this calculation depends on the fusion channel $b$ for the charges $a$ and $x$. The phase can be found from algebraic theory of anyons (Ref. 4) using the diagrams from Fig. 3. The left diagram equals the topological spin $\theta_{b}$, while the right diagram equals $\theta_{a} \theta_{x} \exp \left(i \phi_{a b}\right)$. Hence,

$$
\exp \left(i \phi_{a b}\right)=\frac{\theta_{b}}{\theta_{a} \theta_{x}}
$$

Thus, the tunneling operators can be represented in the form $\hat{X}_{1}=\tilde{X}_{1}, \hat{X}_{2}=\exp \left(i \phi_{\mathrm{AB}}+i \phi_{a b}\right) \tilde{X}_{2}$, where the operators $\tilde{X}_{i}$ are independent of the magnetic flux and the topological charges of the edges.
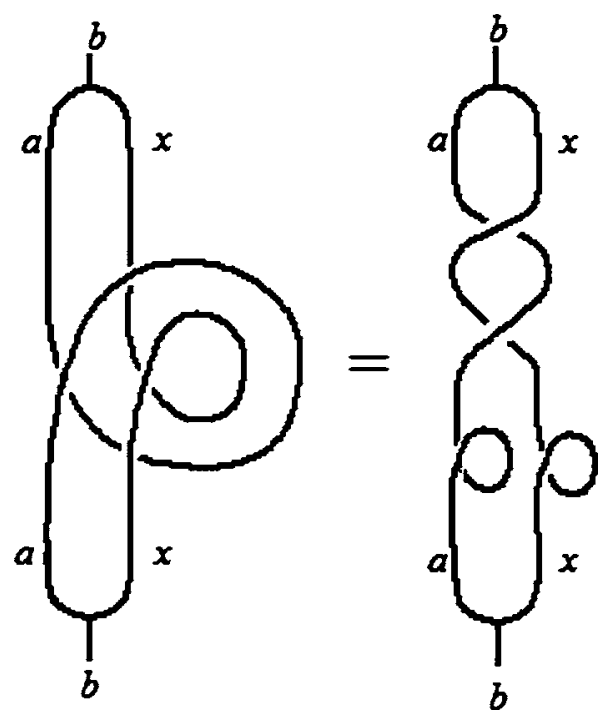

FIG. 3. The diagram for the calculation of the statistical phase $\phi_{a b}$. Note that the left diagram depends only on the combined topological charge $b$ of the excitations $a$ and $x$, which should be viewed as one composite object at the calculation of that diagram.

Since a tunneling event involves a change of the topological charge of edge 2 , we have to find the probabilities of all possible fusion outcomes $b$. The tunneling operators $\tilde{X}_{i}$ create a quasiparticle-quasihole pair, $x$ and $\bar{x}$, near one edge inside a point contact and move the quasiparticle $x$ to the opposite edge. Since the topological charge is an integral of motion, the quasiparticle and quasihole fuse to vacuum. We want to find the amplitude of the process in which $x$ and $a$ fuse to $b$. This amplitude is given by the diagram in Fig. 4 . The vertices are two operators $F_{a x ; i}^{b}$ and $F_{\bar{a} \bar{x} ; j}^{\bar{b}}$ from the fusion space $V_{a x}^{b}$ (Ref. 4); the indices $i$ and $j$ assume values between 1 and the fusion multiplicity $N_{a x}^{b}$. The diagram (Fig. 4) is well known in algebraic theory of anyons. In order to apply the expression from Ref. 4 for this diagram, one just needs to remember that in algebraic theory of anyons, quantum states have norms different from 1 . Thus, up to an irrelevant phase,

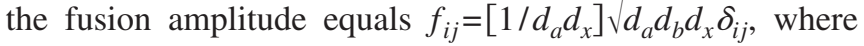
the square bracket contains the normalization factor and $d_{\alpha}$ denote quantum dimensions. Finally, the fusion probability

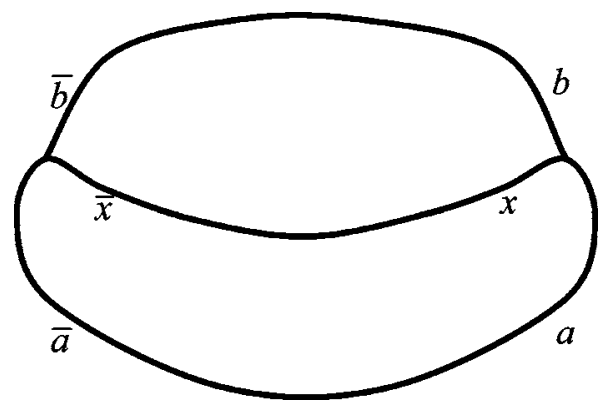

FIG. 4. The diagram for the calculation of the fusion amplitudes. 
TABLE I. Quantum dimensions and topological spins for excitations with different electric and topological charges in the Pfaffian state.

\begin{tabular}{lccc}
\hline \hline $\begin{array}{l}\text { Topological } \\
\text { charge }\end{array}$ & $\begin{array}{c}\text { Electric } \\
\text { charge }\end{array}$ & $\begin{array}{c}\text { Quantum } \\
\text { dimension }\end{array}$ & $\begin{array}{c}\text { Topological } \\
\text { spin }\end{array}$ \\
\hline 1 & $n e / 2$ & 1 & $\exp \left(i \pi n^{2} / 2\right)$ \\
$\epsilon$ & $n e / 2$ & 1 & $-\exp \left(i \pi n^{2} / 2\right)$ \\
$\sigma$ & $e / 4+n e / 2$ & $\sqrt{2}$ & $\exp \left(i \pi\left[2 n^{2}+2 n+1\right] / 4\right)$ \\
\hline \hline
\end{tabular}

$$
P_{a+x \rightarrow b}^{+}=\sum_{i j}\left|f_{i j}\right|^{2}=N_{a x}^{b} \frac{d_{b}}{d_{a} d_{x}} .
$$

The total transition rate equals

$$
w_{a+x \rightarrow b}^{+}=P_{a+x \rightarrow b}^{+} u_{a+x \rightarrow b}^{+},
$$

where $u_{a+x \rightarrow b}^{+}$is the transition rate in the fusion channel $b$. We consider fully absorbing leads. Hence, the rate $u_{a+x \rightarrow b}^{+}$ depends on the previous tunneling events only through the factor $\exp \left(i \phi_{a b}\right)$ in the operator $\hat{X}_{2}$. The rate can be found from Fermi's golden rule:

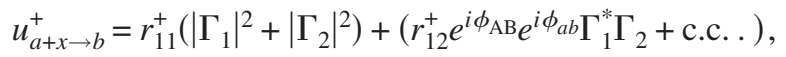

where $r_{\alpha \beta}^{+}=\int_{-\infty}^{\infty}\left\langle\tilde{X}_{\alpha}^{\dagger}(t) \tilde{X}_{\beta}(0)\right\rangle / \hbar^{2}$, and the angular brackets denote the average with respect to the thermodynamic state of noninteracting edges at the temperature $T$ and the chemical potential difference $q V$ between the edges. The correlation functions $r_{\alpha \beta}^{+}$depend on microscopic details.

At finite temperatures, quasiparticles can also tunnel from internal edge 2 to edge 1 , whose electrochemical potential is higher. The respective transition rates

$$
w_{b \rightarrow a+x}^{-}=P_{b \rightarrow a+x}^{-} u_{b \rightarrow a+x}^{-}
$$

can be found in exactly the same way as $w^{+}$. In full analogy with Eq. (14), the diagram from Fig. 4 with interchanged $b \leftrightarrow \bar{a}$ and $\bar{b} \leftrightarrow a$ yields

$$
P_{b \rightarrow a+x}^{-}=N_{a x}^{b} \frac{d_{a}}{d_{b} d_{x}} .
$$

The transition rate $u_{b \rightarrow a+x}^{-}$within the fusion channel $b \rightarrow a$ $+x$ is related to $u_{a+x \rightarrow b}^{+}$by the detailed balance condition

$$
u_{b \rightarrow a+x}^{-}=\exp (-q V / T) u_{a+x \rightarrow b}^{+} .
$$

Equations (14)-(19) allow one to easily reproduce Eqs. (5) and (8) from the previous section. One just needs to know the quantum dimensions, topological spins, and fusion multiplicities. In Laughlin states, all quantum dimensions and fusion multiplicities are 1. The topological spin of the excitation with the electric charge nev equals $\exp \left(i \nu n^{2}\right)$, where $\nu$ is the filling factor. The topological spins and quantum dimension in the Pfaffian states are listed in Table I (cf. Refs. $\left.{ }^{4,14,16}\right)$. All fusion multiplicities $N_{a x}^{b}=1$.

\section{FINITE-TEMPERATURE CURRENT}

Now we are in the position to calculate the tunneling current through the interferometer. We consider the most general situation when the tunneling of several types of quasiparticles with electric charges $k q$ is allowed ( $k$ are integers). Our final answer [Eq. (28)] reproduces the results of Sec. II in the limit of zero temperature for the Laughlin and Pfaffian states.

Let $Q(t)$ be the total charge of edge 2. Then the current

$$
I=\lim _{t \rightarrow \infty}[Q(t)-Q(0)] / t \text {. }
$$

The charge $Q(t)$ can be found from the generating function $q(z, t)=q \sum p_{a, n q} z^{n}$, where $p_{a, n q}$ is the probability to find topological charge $a$ and electric charge $n q$ on edge 2. One gets

$$
Q=\left.\frac{d}{d z} q(z, t)\right|_{z=1}
$$

The probabilities $p_{a, n q}$ obey the kinetic equations

$$
\dot{p}_{a, n q}=\sum_{b, m q} M_{b, m q}^{a, n q} p_{b, m q},
$$

where the kinetic matrix

$$
\begin{aligned}
M_{b, m q}^{a, n q}= & \sum_{x}\left[w_{(b+x), m \rightarrow a, n}^{+}+w_{b, m \rightarrow(a+x), n}^{-}\right] \\
& -\delta_{a b} \delta_{n m} \sum_{c, l, x}\left[w_{(a+x), n \rightarrow c, k}^{+}+w_{a, n \rightarrow(c+x), k}^{-}\right] .
\end{aligned}
$$

The diagonal elements of the kinetic matrix involve infinite sums, but only few contributions to these sums are relevant since the tunneling of quasiparticles $x$ with high electric charges $q(k-n)$ is suppressed.

The system (22) includes an infinite number of equations. It is possible to reduce it to a system of finite size using a symmetry of the kinetic matrix. Indeed, the matrix elements $M_{b, m q}^{a, n q}$ depend on the final and initial topological charges $b$ and $a$ of edge 2 and on the electric charge of the transmitted quasiparticle $q(n-m)$, but they are independent of the initial electric charge $m q$ of edge 2 . Let us introduce a set of generating functions $p_{a}(z, t)=\Sigma_{n} p_{a, n q} z^{n}$, where the summation extends over all states of edge 2 with the same topological charge $a$. The kinetic equation (22) can be rewritten as

$$
\dot{p}_{a}(z)=\sum_{b} K_{b}^{a}(z) p_{b}(z),
$$

where $K_{b}^{a}(z)=\Sigma_{m} M_{b, m q}^{a, n q} z^{n-m}$ and $n$ is arbitrary. The system (24) contains a finite number of equations.

A general solution of Eq. (24) is expressed via the eigenvalues of the matrix $\hat{K}(z)=K_{b}^{a}(z)$. At $z=1$, the matrix satisfies the conditions of the Rorbach theorem: ${ }^{27}$ All its elements are real, all diagonal elements are negative, all nondiagonal elements are positive or zero, and the sum of the elements in each column is zero. Hence, at $z=1$, its maximal eigenvalue is nondegenerate and equals zero. At $z$ close to 1 , the maximal eigenvalue must be close to zero and still be nondegenerate. This strongly simplifies the solution of Eq. (24) at large times $t$. One finds that $p_{a}(z, t)=p_{a}^{(0)}(z) \exp [\lambda(z) t]$, where $\lambda(z)$ is the maximal eigenvalue of the matrix $\hat{K}(z)$. The con- 
servation of probability means that $\Sigma_{a} p_{a}(z=1, t)=1$. At large $t$, this yields $\Sigma_{a} p_{a}^{(0)}(z=1)=1$.

We are finally ready to calculate the current (20) and (21). One obtains

$$
\begin{aligned}
Q= & \left.q \frac{d}{d z} \sum_{a} p_{a}\right|_{z=1, t}=q \lambda^{\prime}(z=1) t \sum_{a} p_{a}^{(0)}(z=1) \\
& +q \sum_{a} \frac{d p_{a}^{(0)}(z=1)}{d z}=q t \lambda^{\prime}(z=1)+q \sum_{a} \frac{d p_{a}^{(0)}(z=1)}{d z},
\end{aligned}
$$

$$
I=q \lambda^{\prime}(z=1) .
$$

Thus, in order to calculate the current, one only needs the eigenvalue $\lambda(z)$ of the matrix $\hat{K}(z)$. We would like to emphasize again that this matrix is finite. The above Eq. (26) can be represented in the computationally more convenient form. Let $\langle\eta|=(1,1, \ldots, 1)$, let

$$
\hat{K}(z)|v(z)\rangle=\lambda(z)|v(z)\rangle,
$$

and let $\langle\eta \mid v\rangle=1$. Note that $\langle\eta| \hat{K}(1)=0$. Differentiating Eq. (27) with respect to $z$ yields $\lambda^{\prime}|v\rangle+\lambda\left|v^{\prime}\right\rangle=\hat{K}^{\prime}|v\rangle+\hat{K}\left|v^{\prime}\right\rangle$. Multiplying by $\langle\eta|$ and setting $z=1$ produces the final result:

$$
I / q=\lambda^{\prime}(1)=\left\langle\eta\left|\hat{A}^{(1)}\right| v\right\rangle,
$$

where $\hat{A}^{(s)}=(z d / d z)^{s} \hat{K}(z=1)$.

\section{FINITE-TEMPERATURE NOISE AT LOW FREQUENCY}

The purpose of this section consists in the calculation of the noise at arbitrary temperature for arbitrary statistics. The results provide an independent check for Sec. II in the limit of zero temperature. They complement the results for the tunneling probabilities and the current from Secs. III and IV. The coefficients $r_{i j}^{+}$in expression (16) for the tunneling rates depend on unknown microscopic parameters and can be extracted by fitting experimental data with the expression for the current [Eq. (28)]. The same information can be independently obtained from the noise [Eq. (34)].

The calculation of the effective charge $e^{*}$ in the lowfrequency limit is based on Eq. (6). We already found the average transmitted charge $Q$ [Eq. (25)]. We need to find $\overline{\delta Q^{2}}=\overline{Q^{2}}-\bar{Q}^{2}$, where $\bar{Q}=q t \lambda^{\prime}(1)+q \sum d p_{a}^{(0)} / d z$ [Eq. (25)]. The generating function method yields the following expression for $\overline{Q^{2}}$ :

$$
\begin{aligned}
\overline{Q^{2}}= & \left.q^{2} \frac{d}{d z} z \frac{d}{d z} \sum_{a} p_{a}\right|_{z=1}=q^{2}\left\{\left[\lambda^{\prime}(1)\right]^{2} t^{2}+\left[\lambda^{\prime \prime}(1)+\lambda^{\prime}(1)\right] t\right. \\
& \left.+2 \lambda^{\prime}(1) t \sum_{a} \frac{d p_{a}^{(0)}}{d z}+\sum_{a}\left[\frac{d}{d z}+\frac{d^{2}}{d z^{2}}\right] p_{a}^{(0)}\right\} .
\end{aligned}
$$

This gives the following answer for the effective charge:

$$
e^{*}=q\left[1+\lambda^{\prime \prime}(1) / \lambda^{\prime}(1)\right] .
$$

It is convenient to express the above result via the matrices $A^{(s)}$ [Eq. (28)]. Differentiating Eq. (27) twice yields

$$
\hat{K}|v\rangle+2 \hat{K}\left|v^{\prime}\right\rangle+\hat{K}\left|v^{\prime \prime}\right\rangle=\lambda^{\prime \prime}|v\rangle+2 \lambda^{\prime}\left|v^{\prime}\right\rangle+\lambda\left|v^{\prime \prime}\right\rangle .
$$

Substituting $z=1$ and multiplying by $\langle\eta|$ gives

$$
\begin{aligned}
\lambda^{\prime \prime}(1)= & \langle\eta|\hat{K}| v\rangle+2\left\langle\eta|\hat{K}| v^{\prime}(1)\right\rangle=\left\langle\eta\left|\hat{A}^{(2)}-\hat{A}^{(1)}\right| v\right\rangle+2\langle\eta| \hat{A}^{(1)} \\
& \times\left|v^{\prime}\right\rangle,
\end{aligned}
$$

where we used the identity $\left\langle\eta \mid v^{\prime}\right\rangle=[\langle\eta \mid v\rangle]^{\prime}=0$. In order to complete the calculation, we need to find $\left|v^{\prime}(1)\right\rangle$. At $z=1$, we can rewrite the $z$ derivative of Eq. (27) in the form $\hat{K}\left|v^{\prime}\right\rangle=$ $-\left(K^{\prime}-\lambda^{\prime} \hat{I}\right)|v\rangle=-(\hat{I}-|v\rangle\langle\eta|) \hat{A}^{(1)}|v\rangle$, where we substituted expression (28) for $\lambda^{\prime}$. Let us now introduce the pseudoinverse matrix $\widetilde{K}^{-1}$ such that $\hat{K} \widetilde{K}^{-1}=\widetilde{K}^{-1} \hat{K}=\hat{I}-|v\rangle\left\langle\eta\left|, \widetilde{K}^{-1}\right| v\right\rangle=0$ and $\langle\eta| \tilde{K}^{-1}=0$. We find

$$
\begin{aligned}
\left|v^{\prime}(1)\right\rangle= & (\hat{I}-|v\rangle\langle\eta|)\left|v^{\prime}\right\rangle=-\widetilde{K}^{-1}(\hat{I}-|v\rangle\langle\eta|) \hat{A}^{(1)}|v\rangle=-\widetilde{K}^{-1} \hat{A}^{(1)} \\
& \times|v\rangle .
\end{aligned}
$$

Combining Eqs. (28), (32), and (33), we finally obtain

$$
e^{*}=q \frac{\left\langle\eta\left|\hat{A}^{(2)}-2 \hat{A}^{(1)} \tilde{K}^{-1} \hat{A}^{(1)}\right| v\right\rangle}{\left\langle\eta\left|\hat{A}^{(1)}\right| v\right\rangle} .
$$

One can easily check that the above equation reproduces the results of Sec. II in the Pfaffian and Laughlin cases. In order to apply the results of Secs. IV and V to the Pfaffian state, one has to modify the definition of the topological charge in comparison with Sec. II. The topological charge can take six different values corresponding to the six vertices of the graph in Fig. 2(b). This means that electrons carry the vacuum topological charge. Such a definition is physically sensible since electrons do not acquire statistical phases when they encircle other excitations. ${ }^{28}$

\section{FINITE-TEMPERATURE NOISE AT HIGH FREQUENCY}

In this section, we assume that only one type of quasiparticles can tunnel between the edges. We denote the electric charge of the tunneling quasiparticle as $q$ and its topological charge as $x$. We also assume nondegenerate braiding ${ }^{4}$ for the topological charge $x$ : This means that for some topological charge $a$, a nontrivial statistical phase $\phi_{a b} \neq 2 \pi n$ is accumulated when a particle $x$ makes a circle around $a$.

If only one type of particles is allowed to tunnel, then the expression for the dc simplifies:

$$
I=q \sum_{a, b} v_{a}\left(w_{a+x \rightarrow b}^{+}-w_{a \rightarrow b+x}^{-}\right),
$$


where the tunneling probabilities $w^{ \pm}$can be found in Sec. III, and $v_{a}$ is the probability to find the topological charge $a$ on edge 2. $v_{a}$ can be extracted from the stationary solution of the kinetic equation (22) and are the components of the vector $|v\rangle$ [Eq. (27)].

The expression for the noise (1) can be rewritten as

$S=\frac{1}{4 \Delta t} \int_{-\Delta t}^{\Delta t} d t_{1} \int_{-\infty}^{\infty} d t_{2}\left\langle I\left(t_{1}\right) I\left(t_{2}\right)+I\left(t_{2}\right) I\left(t_{1}\right)\right\rangle \exp \left(i \omega\left[t_{2}-t_{1}\right]\right)$,

where the time $\Delta t$ is much greater than the duration of one tunneling event and much shorter than the inverse frequency $1 / \omega$. Contributions from large times $\left|t_{2}\right|>1 / \omega$ are suppressed by the rapidly oscillating exponential factor. Contributions from the region $\Delta t<\left|t_{2}\right|<1 / \omega$ correspond to rare situations when one tunneling event occurs at the moment of time $t_{1},\left|t_{1}\right|<\Delta t$, and another one at the moment of time $t_{2}$, $\left|t_{2}\right|<1 / \omega$. Neglecting them, we can reduce Eq. (36) to the following form:

$$
\begin{aligned}
S & =\frac{1}{4 \Delta t} \int_{-\Delta t}^{\Delta t} d t_{1} \int_{-\Delta t}^{\Delta t} d t_{2}\left\langle I\left(t_{1}\right) I\left(t_{2}\right)+I\left(t_{2}\right) I\left(t_{1}\right)\right\rangle \\
& =\frac{1}{4 \Delta t} \int_{-\Delta t}^{\Delta t} d t_{1} \int_{-\Delta t}^{\Delta t} d t_{2}\left\langle\frac{d Q\left(t_{1}\right)}{d t} \frac{d Q\left(t_{2}\right)}{d t}+\frac{d Q\left(t_{2}\right)}{d t} \frac{d Q\left(t_{1}\right)}{d t}\right\rangle \\
& =\frac{1}{2 \Delta t}\left\langle[Q(\Delta t)-Q(-\Delta t)]^{2}\right\rangle=\frac{1}{2 \Delta t}\left\langle\Delta Q^{2}\right\rangle
\end{aligned}
$$

where $\Delta Q$ denotes the change of the electric charge of edge 2 after the time interval $2 \Delta t$, and we used the relations $\exp (i \omega \Delta t) \approx 1$ and $I=d Q / d t$. Possible values of $\Delta Q=n q$ correspond to an integer number of transmitted quasiparticles. Neglecting the contributions from large $n,|n|>1$, one finds

$$
S=q^{2} \sum_{a, b} v_{a}\left(w_{a+x \rightarrow b}^{+}+w_{a \rightarrow b+x}^{-}\right) .
$$

This expression can be evaluated on the basis of the kinetic equations discussed in Sec. IV.

The situation considerably simplifies in the thermal equilibrium case, $V=0$, since the distribution function $v_{a}$ can be found from the detailed balance principle. At zero voltage, the tunneling rates $u^{ \pm}$[Eq. (19)] are equal and, hence, $d_{a}^{2} w_{a+x \rightarrow b}^{+}=d_{b}^{2} w_{b \rightarrow a+x}^{-}$. From the detailed balance condition $v_{a} w_{a+x \rightarrow b}^{+}=v_{b} w_{b \rightarrow a+x}^{-}$, we get

$$
v_{a}=\frac{d_{a}^{2}}{D^{2}}
$$

where the normalization constant is the total quantum dimension $D=\sqrt{\sum_{a} d_{a}^{2}}$. The substitution of Eqs. (15), (17), and (39) into Eq. (38) yields

$$
S=S_{1}+S_{2}+S_{2}^{*},
$$

where $S_{2}$ depends on the magnetic flux and $S_{1}$ exhibits no flux dependence:

$$
\begin{gathered}
S_{1}=\frac{2 q^{2}}{d_{x} D^{2}} r_{11}^{+} \sum_{a, b} N_{a x}^{b} d_{a} d_{b}\left[\left|\Gamma_{1}\right|^{2}+\left|\Gamma_{2}\right|^{2}\right], \\
S_{2}=\frac{2 q^{2}}{d_{x} D^{2}} \sum_{a b} N_{a x}^{b} d_{a} d_{b} \frac{\theta_{b}}{\theta_{a} \theta_{x}} r_{12}^{+} \Gamma_{1}^{*} \Gamma_{2} \exp \left(i \phi_{\mathrm{AB}}\right) .
\end{gathered}
$$

The expression for $S_{1}$ can be simplified with the identity $\Sigma_{b} N_{a x}^{b} d_{b}=d_{a} d_{x}$ (Ref. 4). This yields

$$
S_{1}=2 q^{2} r_{11}^{+}\left[\left|\Gamma_{1}\right|^{2}+\left|\Gamma_{2}\right|^{2}\right] \text {. }
$$

It is not surprising that the flux-independent noise is expressed via the fractional charge and contains no information about fractional statistics. Interestingly, the same is true for the total noise $S$. In fact, $S=S_{1}$ and $S_{2}=0$ as can be seen from the following argument. The combination $\frac{1}{D} \Sigma_{b} N_{a x}^{b} d_{b} \frac{\theta_{b}}{\theta_{a} \theta_{x}}$ $=s_{a \bar{x}}=s_{x a}^{*}$ is an element of the topological $S$ matrix. ${ }^{4}$ Hence, $S_{2}=2 q^{2} r_{12}^{+} \Gamma_{1}^{*} \Gamma_{2} \exp \left(i \phi_{\mathrm{AB}}\right) \Sigma_{a} s_{x a}^{*}\left(d_{a} / D\right)=\mathrm{const} \Sigma_{a} s_{x a}^{*} s_{1 a}=0$. In the last equality, we used the orthogonality of the nontrivial fusion character $s_{x a} / s_{x 1}$ and the trivial character $s_{1 a} / s_{11}$. Thus, the equilibrium high-frequency noise is independent of fractional statistics and the magnetic flux through the interferometer and does not exhibit quantum interference effects in the lowest order in $\Gamma$ 's. We would like to emphasize our assumption that the tunneling quasiparticle exhibits nontrivial braiding with other topological charges. Otherwise, $S_{2} \neq 0$. In particular, this means that quantum interference can be observed if electron tunneling is allowed. Note that the equilibrium high-frequency noise [Eqs. (40) and (43)] is related to the linear conductance by the fluctuationdissipation theorem.

\section{CONCLUSIONS}

In conclusion, we have calculated shot noise in the MachZehnder interferometer in fractional QHE states. At zero temperature, the Fano factor is always smaller than 1 in Abelian states and can exceed 1 in the Pfaffian state. Thus, noise in the Mach-Zehnder interferometer can be used for an experimental probe of non-Abelian statistics. An advantage of such a setup consists in the robustness with respect to the fluctuations of the number of the quasiparticles trapped inside the system. Our predictions are independent of the model of the edges. They apply even in the regime of "short edges," where the physics of the edges cannot be separated from the physics of the leads in the relevant energy window. The only assumption about the edges consists in their chirality.

We also obtained general expressions for the current and noise at arbitrary temperatures and voltages for any anyonic statistics. The expressions drastically simplify at zero voltage in the high-frequency regime. In that regime, no quantum interference effects can be observed for any fractional statistics in sharp contrast to the case of fermions.

Our analysis assumes weak quasiparticle tunneling. For 
Fermi and Bose systems, this would mean a single-particle problem. For anyons, many-particle effects persist even for weak tunneling due to the "black hole" in the center of the interferometer (the hole encircled by edge 2 in Fig. 1). The black hole is entangled with the rest of the system and interacts with tunneling topological charges. This results in a rather unusual behavior including nonanalytic dependence of the current and noise on the small tunneling amplitudes. It should be possible to extend our approach to more complicated geometries with several black holes.

\section{ACKNOWLEDGMENTS}

We acknowledge the support by ARO under Grants Nos. W911NF-04-1-0236 (A.K.) and W911NF-05-1-0294 (A.K.), by NSF under Grants Nos. PHY99-07949 (D.E.F.), PHY0456720 (A.K.), and DMR-0544116 (D.E.F. and K.T.L.), by the U.S.-Israel BSF (Y.G. and A.S.), the Minerva Foundation (A.S.), and the ISF of the Israel Academy of Sciences (Y.G.). D.E.F. acknowledges the hospitality of Microsoft Station Q and KITP in Santa Barbara.
${ }^{1}$ G. Moore and N. Read, Nucl. Phys. B 360, 362 (1991).

${ }^{2}$ C. Nayak and F. Wilczek, Nucl. Phys. B 479, 529 (1996).

${ }^{3}$ A. Yu. Kitaev, Ann. Phys. (N.Y.) 303, 2 (2003).

${ }^{4}$ A. Kitaev, Ann. Phys. (N.Y.) 321, 2 (2006).

${ }^{5}$ R. de Picciotto, M. Reznikov, M. Heiblum, V. Umansky, G. Bunin, and D. Mahalu, Nature (London) 389, 162 (1997); L. Saminadayar, D. C. Glattli, Y. Jin, and B. Etienne, Phys. Rev. Lett. 79, 2526 (1997).

${ }^{6}$ C. de C. Chamon, D. E. Freed, S. A. Kivelson, S. L. Sondhi, and X. G. Wen, Phys. Rev. B 55, 2331 (1997).

${ }^{7}$ S. B. Isakov, T. Martin, and S. Ouvry, Phys. Rev. Lett. 83, 580 (1999); I. Safi, P. Devillard, and T. Martin, ibid. 86, 4628 (2001); S. Vishveshwara, ibid. 91, 196803 (2003); E.-A. Kim, M. Lawler, S. Vishveshwara, and E. Fradkin, ibid. 95, 176402 (2005).

${ }^{8}$ C. L. Kane, Phys. Rev. Lett. 90, 226802 (2003).

${ }^{9}$ K. T. Law, D. E. Feldman, and Y. Gefen, Phys. Rev. B 74, 045319 (2006).

${ }^{10}$ E. Grosfeld, S. H. Simon, and A. Stern, Phys. Rev. Lett. 96, 226803 (2006).

${ }^{11}$ E. Fradkin, C. Nayak, A. Tsvelik, and F. Wilczek, Nucl. Phys. B 516, 704 (1998).

${ }^{12}$ S. Das Sarma, M. Freedman, and C. Nayak, Phys. Rev. Lett. 94, 166802 (2005).

${ }^{13}$ A. Stern and B. I. Halperin, Phys. Rev. Lett. 96, 016802 (2006).

${ }^{14}$ P. Bonderson, A. Kitaev, and K. Shtengel, Phys. Rev. Lett. 96, 016803 (2006).

${ }^{15}$ C.-Y. Hou and C. Chamon, Phys. Rev. Lett. 97, 146802 (2006).

${ }^{16}$ D. E. Feldman and A. Kitaev, Phys. Rev. Lett. 97, 186803 (2006).

${ }^{17}$ F. E. Camino, W. Zhou, and V. J. Goldman, Phys. Rev. B 74,
115301 (2006), and references therein; arXiv:cond-mat/0611443 (unpublished).

${ }^{18}$ B. Rosenow and B. I. Halperin, Phys. Rev. Lett. 98, 106801 (2007).

${ }^{19}$ Y. Ji, Y. C. Chung, D. Sprinzak, M. Heiblum, D. Mahalu, and H. Shtrikman, Nature (London) 422, 415 (2003).

${ }^{20}$ S. Das Sarma, M. Freedman, C. Nayak, S. H. Simon, and A. Stern, arXiv:0707.1889 (unpublished).

${ }^{21}$ Note the coefficient $1 / 2$ in our definition.

${ }^{22}$ F. Marquardt and C. Bruder, Phys. Rev. Lett. 92, 056805 (2004); F. Marquardt, Europhys. Lett. 72, 788 (2005); V. S.-W. Chung, P. Samuelsson, and M. Büttiker, Phys. Rev. B 72, 125320 (2005); H. Forster, S. Pilgram, and M. Büttiker, ibid. 72, 075301 (2005); M. Veillette, J. Chalker, and Y. Gefen, arXiv:cond-mat/0703162 (unpublished).

${ }^{23}$ C. L. Kane and M. P. A. Fisher, Phys. Rev. Lett. 72, 724 (1994).

${ }^{24}$ At $\nu=1 / 3$, the quasihole transfer operator is the only relevant operator (Ref. 25). At $\nu=5 / 2$, the operator which transfers electric charge $e / 2$ and topological charge 1 is relevant; the operator which transfers zero electric charge and topological charge $\epsilon$ is marginal (Ref. 26).

${ }^{25}$ C. L. Kane and M. P. A. Fisher, in Perspectives in Quantum Hall Effect, edited by S. Das Sarma and A. Pinczuk (Wiley, New York, 1997).

${ }^{26}$ P. Fendley, M. P. A. Fisher, and C. Nayak, Phys. Rev. B 75, 045317 (2007).

${ }^{27}$ E. B. Fel'dman, Theor. Exp. Chem. 10, 645 (1976).

${ }^{28}$ Indeed, any anyonic system is, in fact, made of electrons. When one electron makes a full circle around any number of other electrons, only the phase of the form $2 \pi n$ can be accumulated. This is equivalent to no phase at all. 